\title{
A Study on Anti Fuzzy Sub-Bigroup
}

\author{
R.Muthuraj \\ Department of Mathematics \\ PSNA CET, Dindigul. - 624622
}

\author{
M.Rajinikannan \\ Department of MCA \\ PSNA CET, Dindigul. - 624622
}

\author{
M.S.Muthuraman \\ Department of Mathematics \\ PSNA CET, Dindigul. - 624622
}

\begin{abstract}
In this paper, we made an attempt to study the algebraic nature of anti fuzzy sub-bigroup of a bigroup and anti fuzzy sub-bigroup of a group and discussed some of its properties with counter example.

2000 Mathematics Subject Classification: 22F05, 06F10.
\end{abstract}

\section{Keywords}

Fuzzy set, anti fuzzy subgroup, anti fuzzy sub-bigroup of a bigroup, anti fuzzy sub-bigroup of a group, bi-lower level subset, homomorphism, anti-homomorphism.

\section{INTRODUCTION}

The concept of fuzzy sets was initiated by Zadeh. Then it has become a vigorous area of research in engineering, medical science, social science, graph theory etc. Rosenfeld gave the idea of fuzzy subgroups and Ranjith Biswas gave the idea of anti fuzzy subgroups.

The notion of bigroup was first introduced by P.L.Maggu in 1994. W.B. Vasantha Kandasamy and D.Meiyappan introduced concept of fuzzy sub-bigroup of a bigroup and fuzzy sub-bigroup of a group.

In this paper, we introduce the concept of anti fuzzy sub-bigroup of a bigroup and bi-lower level subset of an anti fuzzy subbigroup and anti fuzzy sub-bigroup of a group and prove some results with counter examples. Also we establish the relationship between anti fuzzy sub-bigroup of a group and anti fuzzy subgroup of a group.

\section{Preliminaries}

This section contains some definitions and results to be used in the sequel.

\subsection{Definition}

Let $\mathrm{S}$ be a set. A fuzzy subset $\mathrm{A}$ of $\mathrm{S}$ is a function $\mathrm{A}: \mathrm{S} \rightarrow[0,1]$.

\subsection{Definition}

Let $\mathrm{G}$ be a group. A fuzzy subset A of $\mathrm{G}$ is called an anti fuzzy subgroup if for $\mathrm{x}, \mathrm{y} \in \mathrm{G}$,

$$
\begin{aligned}
& \mathrm{A}(\mathrm{xy}) \leq \max \{\mathrm{A}(\mathrm{x}), \mathrm{A}(\mathrm{y})\}, \\
& \mathrm{A}\left(\mathrm{x}^{-1}\right)=\mathrm{A}(\mathrm{x}) .
\end{aligned}
$$

\subsection{Definition}

Let A be a fuzzy subset of S. For $\mathrm{t} \epsilon[0,1]$, the lower level subsets of $\mathrm{A}$ is the set,

$$
\bar{A} t=\{x \in S: A(x) \leq t\} .
$$

\subsection{Definition}

Let $\mathrm{G}$ be a finite group of order $\mathrm{n}$. and $\mathrm{A}$ be an anti fuzzy subgroup of $\mathrm{G}$.

Let $\operatorname{Im}(A)=\{$ ti $: A(x)=$ ti for some $x \in G\}$. Then $\{\bar{A} t i\}$ are the only lower level subgroups of A.

\subsection{Definition}

A set $(\mathrm{G},+, \bullet)$ with two binary operation ' + ' and '• ' is called a bigroup if there exist two proper subsets G1 and G2 of G such that
i. $G=G_{1} \cup G_{2}$
ii. $\left(\mathrm{G}_{1},+\right)$ is a group.
iii. $\left(\mathrm{G}_{2}, \bullet\right)$ is a group.

A non-empty subset $\mathrm{H}$ of a bigroup $(\mathrm{G},+, \bullet)$ is called a subbigroup, if $\mathrm{H}$ itself is a bigroup under ' + ' and ' $\bullet$ ' operations defined on $\mathrm{G}$.

\subsection{Definition}

Let $\mathrm{G}=(\mathrm{G},+, \bullet)$ be a bigroup. Then $\mathrm{A}: \mathrm{G} \rightarrow[0,1]$ is said to be an anti fuzzy sub-bigroup of the bigroup $G$ if there exists two fuzzy subsets $\mathrm{A} 1$ of $\mathrm{G} 1$ and $\mathrm{A} 2$ of G2 such that

$$
\begin{aligned}
& \text { i. } A=A_{1} \cup A_{2} \\
& \text { ii. }\left(A_{1},+\right) \text { is an anti fuzzy subgroup of }\left(G_{1},+\right) \text {. } \\
& \text { iii. }\left(A_{2}, \bullet\right) \text { is a an anti fuzzy subgroup }\left(G_{2}, \bullet\right) \text {. }
\end{aligned}
$$

\subsection{Example}

Let $\mathrm{G} 1=\{0, \mathrm{a}, \mathrm{b}, \mathrm{a}+\mathrm{b}\}$ be a group under the operation ' + ' with $a+a=b+b=0$ and $a+b=b+a$

Let $\mathrm{G} 2=\{1,-1, \mathrm{i},-\mathrm{i}\}$ be a group under the operation $\bullet$. Define $A: G \rightarrow[0,1]$ by 


$$
A(x)=\left\{\begin{array}{l}
0.2 \text { for } x=0 \\
0.3 \text { for } x=a, 1 \\
0.4 \text { for } x=b, a+b,-1 \\
0.5 \text { for } x=i,-i
\end{array}\right.
$$

It is easy to verify that $A$ is an anti fuzzy sub-bigroup of the bigroup $\mathrm{G}$, for we can find $\mathrm{A} 1: \mathrm{G} 1 \rightarrow[0,1]$ by

$$
\mathrm{A}_{1}(\mathrm{x})=\left\{\begin{array}{l}
0.2 \text { for } \mathrm{x}=0 \\
0.3 \text { for } \mathrm{x}=\mathrm{a} \\
0.4 \text { for } \mathrm{x}=\mathrm{b}, \mathrm{a}+\mathrm{b}
\end{array}\right.
$$

and $\mathrm{A} 2: \mathrm{G} 2 \rightarrow[0,1]$ is given by

$$
\mathrm{A}_{2}(\mathrm{x})=\left\{\begin{array}{l}
0.3 \text { for } \mathrm{x}=1 \\
0.4 \text { for } \mathrm{x}=-1 \\
0.5 \text { for } \mathrm{x}=\mathrm{i},-\mathrm{i}
\end{array}\right.
$$

Clearly A is an anti fuzzy sub-bigroup of the bigroup G.

\subsection{Theorem}

Every $\mathrm{t}$ - lower level subsets of an anti fuzzy sub-bigroup A of a bigroup $\mathrm{G}$ need not in general be a sub-bigroup of the bigroup $\mathrm{G}$.

Proof: We prove this by an example.

Take $\mathrm{G}=\{-1,0,1\}$ to be a bigroup under the operation ' + ' and ' $\bullet$ ' where $\mathrm{G} 1=\{0\}$ and $\mathrm{G} 2=\{-1,1\}$ are groups respectively with respect to usual addition and usual multiplication.

Define $A: G \rightarrow[0,1]$

$$
A(x)=\left\{\begin{array}{l}
0.5 \text { for } x=-1,1 \\
0.75 \text { for } x=0
\end{array}\right.
$$

Clearly $(\mathrm{A},+, \bullet)$ is an anti fuzzy sub-bigroup of the bigroup $(\mathrm{G},,+$, -). Now consider the lower level subset $\bar{A} t$ for $t=0.5$ of the anti fuzzy sub-bigroup A

For $\mathrm{t}=0.5$,

$$
\begin{aligned}
\overline{\mathrm{A}}_{\mathrm{t}} & =\{\mathrm{x} \in \mathrm{G}: \mathrm{A}(\mathrm{x}) \leq 0.5\} . \\
\overline{\mathrm{A}}_{\mathrm{t}} & =\{-1,1\} .
\end{aligned}
$$

It is easy to verify that $\{-1,1\}$ is not a sub-bigroup of the bigroup $(\mathrm{G},,+, \bullet)$. Hence the lower level subset Āt for $\mathrm{t}=0.5$ of an anti fuzzy sub-bigroup $\mathrm{A}$ is not a sub-bigroup of the bigroup $(\mathrm{G},,+$, $\bullet)$.

We introduce the notion of bi-lower level subset of an anti fuzzy sub-bigroup.

\subsection{Definition}

Let $\mathrm{G}=(\mathrm{G} 1 \cup \mathrm{G} 2,+, \bullet)$ be a bigroup and $\mathrm{A}=(\mathrm{A} 1 \cup \mathrm{A} 2)$ be an anti fuzzy sub-bigroup of the bigroup $\mathrm{G}$. The bi-lower level subset of the anti fuzzy sub-bigroup A of the bigroup $\mathrm{G}$ is defined as

$$
\overline{\mathrm{A}} \mathrm{t}=\overline{\mathrm{A}} 1 \mathrm{t} \cup \overline{\mathrm{A}} 2 \mathrm{t} \text { for every } \mathrm{t} \epsilon[\max \{\mathrm{A} 1(\mathrm{e} 1), \mathrm{A} 2(\mathrm{e} 2)\}, 1] .
$$

Where e1 denotes the identity element of the group $(\mathrm{G} 1,+)$ and e2 denotes the identity element of the group $(\mathrm{G} 2, \bullet)$.

\section{Remark}

The condition $\mathrm{t} \epsilon[\max \{\mathrm{A} 1(\mathrm{e} 1), \mathrm{A} 2(\mathrm{e} 2)\}, 1]$ is essential for the bi-lower level subset to be a sub-bigroup, for if $\mathrm{t} \notin[\max \{\mathrm{A} 1$ ( e1), A2( e2) \},1], the bi-lower level subset need not in general be a sub-bigroup of the bigroup $G$ which is evident from the following example.

\subsection{Example}

Consider Example 1.1, the bi-lower level subset Ât for $\mathrm{t}=0.2$ of the anti fuzzy sub-bigroup $\mathrm{A}$ is given by $\overline{\mathrm{A}} \mathrm{t}=\{0\}$ which is not a sub-bigroup of the bigroup $\mathrm{G}$. Therefore the bilower level subset Āt for $t=0.2$ is not a sub-bigroup of the bigroup $\mathrm{G}$.

\subsection{Theorem}

Every bi-lower level subset of an anti fuzzy sub-bigroup A of a bigroup $\mathrm{G}$ is a sub-bigroup of the bigroup $\mathrm{G}$.

Proof

Let $\mathrm{A}=(\mathrm{A} 1 \cup \mathrm{A} 2)$ be an anti fuzzy sub-bigroup of a bigroup $\mathrm{G}=$ $(\mathrm{G} 1 \cup \mathrm{G} 2,+, \bullet)$. Consider the bi-lower level subset Āt of an anti fuzzy sub-bigroup A for every $t \in[\max$ $\{\mathrm{A} 1(\mathrm{e} 1), \mathrm{A} 2(\mathrm{e} 2)\}, 1]$, where e1 denotes the identity element of the group ( $\mathrm{G} 1,+)$ and e2 denotes the identity element of the group $(\mathrm{G} 2, \bullet)$. Then $\bar{A} t=\bar{A} 1 \mathrm{t} \cup \overline{\mathrm{A}} 2 \mathrm{t}$ where $\overline{\mathrm{A}} 1 \mathrm{t}$ and $\overline{\mathrm{A}} 2 \mathrm{t}$ are subgroups of G1 and $\mathrm{G} 2$ respectively. Hence by the definition of sub-bigroup $\bar{A} t$ is a sub-bigroup of the bigroup $(\mathrm{G},,+, \bullet)$.

We illustrate the following by an example. 


\subsection{Example}

Let $\mathrm{G}=\{0, \pm 1, \pm \mathrm{i}\}$ is a bigroup with respect to addition modulo 2 and multiplication. Clearly $\mathrm{G} 1=\{0,1\}$ and $\quad \mathrm{G} 2=\{ \pm 1, \pm \mathrm{i}\}$ are groups with respect to addition modulo 2 and multiplication respectively. Define A : $\mathrm{G} \rightarrow[0,1]$ by

$$
A(x)=\left\{\begin{array}{ccc}
0.1 & \text { for } & x=0 \\
0.5 \text { for } & x= \pm 1 \\
0.7 \text { for } & x= \pm i
\end{array}\right.
$$

It is easy to verify that $A$ is an anti fuzzy sub-bigroup of the bigroup $\mathrm{G} s$ there exist two anti fuzzy subgroups A1 : $\mathrm{G} 1 \rightarrow[0,1], \mathrm{A} 2: \mathrm{G} 2 \rightarrow[0,1]$ such that $\mathrm{A}=(\mathrm{A} 1 \cup \mathrm{A} 2)$ where

$$
A_{1}(x)=\left\{\begin{array}{l}
0.1 \text { for } x=0 \\
0.4 \text { for } x=1
\end{array}\right.
$$

and

$$
A_{2}(x)=\left\{\begin{array}{l}
0.5 \text { for } x= \pm 1 \\
0.7 \text { for } x= \pm i
\end{array}\right.
$$

We now calculate the bi-lower level subset $\bar{A} t$ for $t=0.5$,

$\overline{\mathrm{A}}_{\mathrm{t}}=\overline{\mathrm{A}}_{1 \mathrm{t}} \cup \overline{\mathrm{A}}_{2 \mathrm{t}}=\left\{\mathrm{x} \in \mathrm{G}_{1}: \mathrm{A}_{1}(\mathrm{x}) \leq 0.5\right\} \cup\left\{\mathrm{x} \in \mathrm{G}_{2}: \mathrm{A}_{2}(\mathrm{x}) \leq\right.$ $0.5\}$

$$
\begin{aligned}
& =\{0,1\} \cup\{-1,1\} \\
& =\{0, \pm 1\} \\
\bar{A}_{t} & =\{0, \pm 1\}
\end{aligned}
$$

It is easily verified that $\bar{A}_{t}$ is a sub-bigroup of the bigroup $G$.

\subsection{Theorem}

Let $\mathrm{G}$ be a bigroup and A1, A2 be fuzzy subsets of A such that $\mathrm{A}=(\mathrm{A} 1 \cup \mathrm{A} 2)$. The bi-lower level subset Āt of A is a subbigroup of $\mathrm{G}, \mathrm{t} \in[\max \{\mathrm{A} 1(\mathrm{e} 1), \mathrm{A} 2(\mathrm{e} 2)\}, 1]$, where e1 denotes the identity element of G1 and e 2 denotes the identity element of $\mathrm{G} 2$ respectively. Then $\mathrm{A}$ is an anti fuzzy sub-bigroup of a bigroup G.

\section{Proof}

$$
\text { Let } G=\left(G_{1} \cup G_{2}\right) \text { be a bigroup. }
$$

Given that the bi-lower level subset $\bar{A} t=\bar{A} 1 t \cup \bar{A} 2 t$ is a subbigroup of $\mathrm{G}$.

Clearly $\bar{A} 1 \mathrm{t}$ is a subgroup of G1, A1 is an anti fuzzy subgroup of G1.

Clearly $\bar{A} 2 t$ is a subgroup of G2, A2 is an anti fuzzy subgroup of G2.

Hence $\mathrm{A}=(\mathrm{A} 1 \cup \mathrm{A} 2)$ and hence $\mathrm{A}$ is an anti fuzzy sub-bigroup of G.

\subsection{Definition}

Let $\mathrm{A}=(\mathrm{A} 1 \cup \mathrm{A} 2)$ be an anti fuzzy sub-bigroup of a bigroup $\mathrm{G}$ $=(\mathrm{G} 1 \cup \mathrm{G} 2)$. The sub-bigroups Āt, for $\quad \mathrm{t} \in[\max$ $\{\mathrm{A} 1(\mathrm{e} 1), \mathrm{A} 2$ ( e2) $\}, 1]$, where e1 denotes the identity element of G1 and e2 denotes the identity element of G2 respectively, are called bi-lower level sub-bigroups of A.

\subsection{Theorem}

Let $\mathrm{A}=(\mathrm{A} 1 \cup \mathrm{A} 2)$ be an anti fuzzy sub-bigroup of a bigroup $\mathrm{G}$ $=(\mathrm{G} 1 \cup \mathrm{G} 2)$. Two bi-lower level subgroups $\bar{A} \alpha, \bar{A} \beta, \alpha, \beta \in[$ $\max \{\mathrm{A} 1(\mathrm{e} 1), \mathrm{A} 2(\mathrm{e} 2)\}, 1]$, where e1 denotes the identity element of $\mathrm{G} 1$ and $\mathrm{e} 2$ denotes the identity element of $\mathrm{G} 2$ respectively with $\alpha<\beta$ are equal iff there is no $\mathrm{x}$ in $\mathrm{G}$ such that $\alpha<\mathrm{A}(\mathrm{x}) \leq \beta$.

\section{Proof}

Let $\mathrm{A}=(\mathrm{A} 1 \cup \mathrm{A} 2)$ be an anti fuzzy sub-bigroup of a bigroup $\mathrm{G}=(\mathrm{G} 1 \cup \mathrm{G} 2)$.

Consider the two bi-lower level subgroups A $\alpha, \overline{\mathrm{A}} \beta, \quad \alpha, \beta$ $\in[\max \{\mathrm{A} 1(\mathrm{e} 1), \mathrm{A} 2(\mathrm{e} 2)\}, 1]$ where e1 denotes the identity element of $\mathrm{G} 1$ and $\mathrm{e} 2$ denotes the identity element of $\mathrm{G} 2$ respectively with $\alpha<\beta$.

Let $\overline{\mathrm{A}}_{\alpha}=\overline{\mathrm{A}}_{\beta}$.

We have to prove that there is no $\mathrm{x}$ in $\mathrm{G}$ such that

$\alpha<\mathrm{A}(\mathrm{x}) \leq \beta$.

Suppose that there is an $\mathrm{x}$ in $\mathrm{G}$ such that $\alpha<\mathrm{A}(\mathrm{x})<\beta$ then $\mathrm{x} \in \overline{\mathrm{A}} \beta$ and $\mathrm{x} \notin \overline{\mathrm{A}} \alpha$.

This implies $\bar{A} \alpha \subset \bar{A} \beta$, which contradicts the assumption that $\overline{\mathrm{A}} \alpha=\overline{\mathrm{A}} \beta$.

Hence there is no $\mathrm{x}$ in $\mathrm{G}$ such that $\alpha<\mathrm{A}(\mathrm{x}) \leq \beta$.

Conversely, suppose that there is no $\mathrm{x}$ in $\mathrm{G}$ such that

$\alpha<\mathrm{A}(\mathrm{x}) \leq \beta$.

Then, by definition, $\bar{A} \alpha \subset \bar{A} \beta$.

Let $\mathrm{x} \in \overline{\mathrm{A}} \beta$ and there is no $\mathrm{x}$ in $\mathrm{G}$ such that $\alpha<\mathrm{A}(\mathrm{x}) \leq \beta$.

Hence $\mathrm{x} \in \overline{\mathrm{A}} \alpha$.

(i.e) $\overline{\mathrm{A}} \beta \subset \overline{\mathrm{A}} \alpha$.

Hence $\overline{\mathrm{A}} \alpha=\overline{\mathrm{A}} \beta$.

\subsection{Theorem}


A fuzzy subset $A$ of $G$ is an anti fuzzy sub-bigroup of $G$ iff the bilower level subsets Āt, $\mathrm{t} \in$ Image A, are sub-bigroups of $\mathrm{G}$.

\section{Proof}

It is clear.

\subsection{Theorem}

Any sub-bigroup $\mathrm{H}$ of a bigroup $\mathrm{G}$ can be realized as a bi-lower level sub-bigroup of some anti fuzzy sub-bigroup of a bigroup $\mathrm{G}$.

Proof: Let $\mathrm{G}=(\mathrm{G} 1 \cup \mathrm{G} 2,+, \bullet)$ be a bigroup.

Let $\mathrm{H}=(\mathrm{H} 1 \cup \mathrm{H} 2,+, \bullet)$ be a sub-bigroup of $\mathrm{G}$.

Let $\mathrm{A} 1$ and $\mathrm{A} 2$ be a fuzzy subsets of A defined by

$A_{1}(x)=\left\{\begin{array}{c}0 \text { if } x \in \mathrm{H}_{1} \\ \mathrm{t} \text { if } \mathrm{x} \notin \mathrm{H}_{1}\end{array} ; \quad A_{2}(x)=\left\{\begin{array}{l}0 \text { if } \mathrm{x} \in \mathrm{H}_{2} \\ \mathrm{t} \text { if } \mathrm{x} \notin \mathrm{H}_{2}\end{array}\right.\right.$

where $\mathrm{t} \in[\max \{\mathrm{A} 1(\mathrm{e} 1), \mathrm{A} 2(\mathrm{e} 2)\}, 1]$, and e1 denotes the identity element of $\mathrm{G} 1$ and e2 denotes the identity element of G2 respectively.

We shall prove that $\mathrm{A}=(\mathrm{A} 1 \cup \mathrm{A} 2)$ is an anti fuzzy sub-bigroup of a bigroup $\mathrm{G}$.

Suppose $\mathrm{x}, \mathrm{y} \in \mathrm{H}$, then

i. $\quad \mathrm{x}, \mathrm{y} \in \mathrm{H}_{1} \Rightarrow \mathrm{x}+\mathrm{y} \in \mathrm{H}_{1}$ and $\mathrm{x}+(-\mathrm{y}) \in \mathrm{H}_{1}$.

$\mathrm{A}_{1}(\mathrm{x})=0, \mathrm{~A}_{1}(\mathrm{y})=0$ and $\mathrm{A}_{1}(\mathrm{x}+(-\mathrm{y}))=0$, then

$A_{1}(x+(-y)) \leq \max \left\{A_{1}(x), A_{1}(y)\right\}$.

ii. $\quad \mathrm{x}, \mathrm{y} \in \mathrm{H}_{2} \Rightarrow \mathrm{xy} \in \mathrm{H}_{2}$ and $\mathrm{xy}^{-1} \in \mathrm{H}_{2}$.

$\mathrm{A}_{2}(\mathrm{x})=0, \mathrm{~A}_{2}(\mathrm{y})=0$ and $\mathrm{A}_{2}\left(\mathrm{xy}^{-1}\right)=0$,

Then $A_{2}\left(x y^{-1}\right) \leq \max \left\{A_{2}(x), A_{2}(y)\right\}$

iii. $\quad \mathrm{x} \in \mathrm{H}_{1}$ and $\mathrm{y} \notin \mathrm{H}_{1} \quad \Rightarrow \mathrm{x}+\mathrm{y} \notin \mathrm{H}_{1}$

and $\mathrm{x}+(-\mathrm{y}) \notin \mathrm{H}_{1}$.

$\mathrm{A}_{1}(\mathrm{x})=0, \mathrm{~A}_{1}(\mathrm{y})=\mathrm{t}$ and $\mathrm{A}_{1}(\mathrm{x}+(-\mathrm{y}))=\mathrm{t}$, then

$A_{1}(x+(-y)) \leq \max \left\{A_{1}(x), A_{1}(y)\right\}$.

iv. $\quad \mathrm{x} \in \mathrm{H}_{2}$ and $\mathrm{y} \notin \mathrm{H}_{2} \quad \Rightarrow \mathrm{xy} \notin \mathrm{H}_{2}$ and $\mathrm{xy}^{-1} \notin \mathrm{H}_{2}$.

$\mathrm{A}_{2}(\mathrm{x})=0, \mathrm{~A}_{2}(\mathrm{y})=\mathrm{t}$ and $\mathrm{A}_{2}\left(\mathrm{xy}^{-1}\right)=\mathrm{t}$,

Then $A_{2}\left(x y^{-1}\right) \leq \max \left\{A_{2}(x), A_{2}(y)\right\}$.

Suppose $\mathrm{x}, \mathrm{y} \notin \mathrm{H}$, then

i. $\quad \mathrm{x}, \mathrm{y} \notin \mathrm{H}_{1}$ then $\mathrm{x}+\mathrm{y} \in \mathrm{H}_{1}$ or $\mathrm{x}+\mathrm{y} \notin \mathrm{H}_{1}$.

$\mathrm{x}, \mathrm{y} \notin \mathrm{H}_{1}$ then $\mathrm{x}+(-\mathrm{y}) \in \mathrm{H}_{1}$ or $\mathrm{x}+(-\mathrm{y}) \notin \mathrm{H}_{1}$.

$\mathrm{A}_{1}(\mathrm{x})=\mathrm{t}, \mathrm{A}_{1}(\mathrm{y})=\mathrm{t}$ and $\mathrm{A}_{1}(\mathrm{x}+(-\mathrm{y}))=0$ or $\mathrm{t}$,

then $A_{1}(x+(-y)) \leq \max \left\{A_{1}(x), A_{1}(y)\right\}$. ii. $\quad \mathrm{x}, \mathrm{y} \notin \mathrm{H}_{2} \Rightarrow \mathrm{xy} \in \mathrm{H}_{2}$ or $\quad \mathrm{xy} \notin \mathrm{H}_{2}$.

$\mathrm{x}, \mathrm{y} \notin \mathrm{H}_{2} \Rightarrow \mathrm{xy}^{-1} \in \mathrm{H}_{2}$ or $\quad \mathrm{xy}^{-1} \notin \mathrm{H}_{2}$.

$\mathrm{A}_{2}(\mathrm{x})=\mathrm{t}, \mathrm{A}_{2}(\mathrm{y})=\mathrm{t}$ and $\mathrm{A}_{2}\left(\mathrm{xy}^{-1}\right)=0$ or $\mathrm{t}$,

then $A_{2}\left(x^{-1}\right) \leq \max \{A 2(x), A 2(y)\}$.

Thus in all cases,

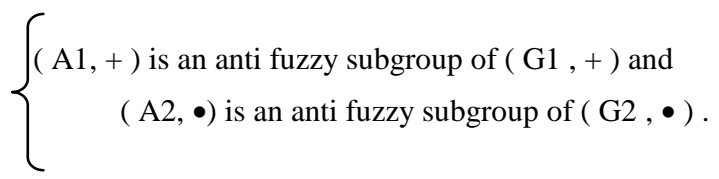

Hence $\mathrm{A}=(\mathrm{A} 1 \cup \mathrm{A} 2)$ is an anti fuzzy sub-bigroup of a bigroup G. For this anti fuzzy sub-bigroup, Āt $=\bar{A} 1 \mathrm{t} \cup \overline{\mathrm{A}} 2 \mathrm{t}=\mathrm{H}$.

\section{Remark}

As a consequence of the above Theorems, the bi-lower level subbigroups of an anti fuzzy sub-bigroup A form a chain. Since A(e1) $\leq \mathrm{A}(\mathrm{x})$ for all $\mathrm{x}$ in $\mathrm{G} 1$ or $\mathrm{A}(\mathrm{e} 2) \leq \mathrm{A}(\mathrm{x})$ for all $\mathrm{x}$ in $\mathrm{G} 2$, therefore $\overline{\mathrm{A}} \mathrm{t} 0$, where $\mathrm{t} 0=\max \{\mathrm{A} 1(\mathrm{e} 1), \mathrm{A} 2(\mathrm{e} 2)\}, \quad$ is the smallest and we have the chain :

$\{\mathrm{e} 1, \mathrm{e} 2\}=\overline{\mathrm{A}} \mathrm{t} 0 \subset \overline{\mathrm{A}} \mathrm{t} 1 \subset \overline{\text { At}} \mathrm{t} 2 \subset \ldots . \subset \overline{\text { Atn }}=\mathrm{G}$, where t0 $<$ t $1<\mathrm{t} 2<\ldots . .<$ tn.

2. Now we proceed on to define the anti fuzzy bigroup of a group.

2.1 Definition

A fuzzy subset A of a group $G$ is said to be an anti fuzzy subbigroup of the group $\mathrm{G}$ if there exist two anti fuzzy subgroups $\mathrm{A} 1$ and $\mathrm{A} 2$ of $\mathrm{A}(\mathrm{A} 1 \neq \mathrm{A}$ and $\mathrm{A} 2 \neq \mathrm{A})$ such that $\mathrm{A}=\mathrm{A} 1 \cup$ A2 .

\subsection{Theorem}

Every anti fuzzy sub-bigroup of a group $G$ is an anti fuzzy subgroup of a group $\mathrm{G}$ but not conversely.

\section{Proof}

It is clear. Since the union of any two anti fuzzy subgroup is an anti fuzzy subgroup.

Converse part is not true in general and is proved by the following example.

\subsection{Example}

Consider the group $\mathrm{G}=\{1,-1, \mathrm{i},-\mathrm{i}\}$ under usual multiplication. Define $A: G \rightarrow[0,1]$ by

$$
A(x)=\left\{\begin{array}{l}
0 \text { for } x=\{1,-1\} \\
1 \text { for } x=\{i,-i\}
\end{array}\right.
$$

Clearly A is an anti fuzzy subgroup as all of its lower level subsets are subgroups of $\mathrm{G}$. Further $\mathrm{o}(\operatorname{Im}(\mathrm{A}))=2$. 
If $\mathrm{Ak}$ is an anti fuzzy subgroup of $\mathrm{A}$ such that $\mathrm{Ak}$ is a subset of A. $(\mathrm{Ak} \neq \mathrm{A})$. Then $\mathrm{Ak}$ takes following form.

$$
A_{k}(x)=\left\{\begin{array}{l}
0 \text { for } x=\{1,-1\} \\
\alpha_{k} \text { for } x=\{i,-i\}
\end{array}\right.
$$

with $0 \leq \alpha_{\mathrm{k}}<1$ for every $\mathrm{i} \in \mathrm{I}$.

It is easy to verify that $\mathrm{Aj} \cup \mathrm{Ak} \neq \mathrm{A}$ for any $\mathrm{j}, \mathrm{k} \in \mathrm{I}$. Thus there does not exist two anti fuzzy subgroups $\mathrm{A} 1$ and $\mathrm{A} 2$ of $\mathrm{A}$ ( $\mathrm{A} 1 \neq$ $\mathrm{A}$ and $\mathrm{A} 2 \neq \mathrm{A}$ ) such that $\mathrm{A}=\mathrm{A} 1 \cup \mathrm{A} 2$

\subsection{Theorem}

Let $A$ be an anti fuzzy subgroup of a group $\mathrm{G}$ with $3 \leq \mathrm{o}(\operatorname{Im}(\mathrm{A}))$ $<\infty$ then there exist two anti fuzzy subgroups A1 and A2 of A $(\mathrm{A} 1 \neq \mathrm{A}$ and $\mathrm{A} 2 \neq \mathrm{A})$ such that $\mathrm{A}=\mathrm{A} 1 \cup \mathrm{A} 2$

\section{Proof}

Let $\mathrm{A}$ be an anti fuzzy subgroup of a group G. Suppose $\operatorname{Im}(\mathrm{A})=$ $\{\mathrm{a} 1, \mathrm{a} 2, \ldots$ an $\}$ with $\mathrm{a} 1<\mathrm{a} 2<\ldots<$ an and $3 \leq \mathrm{n}<\infty$.

Choose b1, b2 $\epsilon\left[\begin{array}{ll}0 & 1\end{array}\right]$ be such that $\mathrm{b} 1 \leq \mathrm{a} 1<\mathrm{b} 2<\mathrm{a} 2<\ldots<$ an and define A1, A2: G $\rightarrow[0,1]$ by,

$$
A_{1}(x)= \begin{cases}a_{1} & \text { for } x \in \bar{A}_{a 1} \\ b_{2} & \text { for } x \in \bar{A}_{a 2}-\bar{A}_{a 1} \\ A(x) & \text { otherwise }\end{cases}
$$

and

$$
A_{2}(x)= \begin{cases}b_{1} & \text { for } x \in \bar{A}_{a 1} \\ a_{2} & \text { for } x \in \bar{A}_{a 2}-\bar{A}_{a 1} \\ A(x) & \text { otherwise }\end{cases}
$$

Clearly $A_{1}$ and $A_{2}$ are anti fuzzy subgroups.

\subsection{Theorem}

Let $A$ be a fuzzy subset of a group $G$ with $3 \leq o(\operatorname{Im}(A))<\infty$. Then $A$ is an anti fuzzy subgroup of the group $G$ iff $A$ is an anti fuzzy sub-bigroup of $\mathrm{G}$.

\section{Proof}

It is clear from Theorem 2.1 and Theorem 2.2

\section{References}

[1] Biswas .R, Fuzzy subgroups and Anti Fuzzy subgroups, Fuzzy sets and Systems, 35(1990) 121-124.

[2] Das. P.S, Fuzzy groups and level subgroups, J.Math.Anal.
Appl, 84 (1981) 264-269.

[3] Maggu, P.L., On introduction of Bigroup concept with its applications in industry, Pure App. Math Sci., 39, 171-173 (1994).

[4] Mohamed Asaad, Groups and Fuzzy subgroups Fuzzy sets and systems 39 (1991) 323-328.

[5] Palaniappan.N , Muthuraj.R , Anti Fuzzy Group and Lower level subgroups, Antarctica J.Math., 1( 1 )(2004), 71 - 76.

[6] Prabir Bhattacharya, Fuzzy Subgroups: Some Characterizations, J.Math. Anal. Appl.128 (1987) 241 - 252.

[7] Rosenfeld, Fuzzy groups, J.Math.Anal.Appl, 35 (1971) $512-517$.

[8] P.Sundararajan , N.Palaniappan , R.Muthuraj , Anti M- Fuzzy subgroup and anti M-Fuzzy sub-bigroup of an M-group , Antartica J.Math., 6(1)(2009), 33-37.

[9] R.Muthuraj, P.M.Sithar Selvam, M.S.Muthuraman, Anti Qfuzzy group and its lower Level subgroups, International journal of Computer Applications (0975-8887),Volume 3no.3, June 2010, 16-20.

[10] R.Muthuraj, M.Sridharan ,M.S.Muthuraman and P.M.Sitharselvam, Anti Q-fuzzy BG-idals in BG-Algebra, International journal of Computer Applications (09758887), Volume 4, no.11, August 2010, 27-31.

[11] P.Sundararajan, R.Muthuraj, Anti M-fuzzy subgroup and its lower level M-subgroups, International Journal of Computer Applications, (0975 -8887), Vol.23, No.3, 32- 35, July 2011.

[12] P.Sundararajan , R.Muthuraj , Anti M-fuzzy sub-bigroup and its bi lower level M-sub bigroups, International Journal of Computer Applications, (0975 -8887 ), Vol.26, No.8, 1- 4, July 2011.

[13] Vasantha Kandasamy, W. B. and Meiyappan, D., Bigroup and Fuzzy bigroup, Bol. Soc. Paran Mat, 18, 59-63 (1998).

[14] Vasantha Kandasamy W. B., "Bialgebraic Structures and Smarandache Bialgebraic Structures “,American Research press, Rehoboth, 2003. 